\title{
Zur elektrokinetischen Sanierung arsenkontaminierter Böden im Land Brandenburg
}

\author{
Udo Hellwig, Olga Böhme, Werner Schmitz, Edgar Wetzig
}

\section{Teil 1: Das Element Arsen im Boden}

Udo Hellwig (ITVA), Olga Böhme

\subsection{Einführung}

Das Grundstuick der Technischen Fachhochschule Wildau, Standort Friedrich-Engels-Straße, und anrainende Grundstücke sind ein alter ehemaliger chemischer Industriestandort, auf dem schon vor dem Ersten Weltkrieg chemische Produkte hergestellt wurden. Die oberirdischen Anlagen sind bis auf wenige Gebäude nicht mehr vorhanden. Unterirdisch sind dagegen noch einige Lager- und Leitungselemente und eine erhebliche Abraummenge vorzufinden, die ein gewisses umweltrelevantes Gefährdungspotenzial darstellen.

Über den Altschaden in Wildau liegen mehrere Erkundungen und Gutachten vor [1]. Es wurden u. a. geoelektrische Erkundungen, eine elektromagnetische Kartierung, Grundwasser- und Bodenbeprobungen und -analysen durchgefuihrt. Schwerpunkt der Kontamination ist neben Blei der hohe Arsengehalt in Boden und Grundwasser des Geländes. Quellen der Belastung mit Arsen und Schwermetallen auf dem Gelände der Technischen Fachhochschule Wildau (TFH Wildau) sind die ehemalige Schwefelsäureherstellung und Sulfurylchlorid-Anlage.

Auf dem ca. $2.000 \mathrm{~m}^{2}$ großen Gelände wurden zahlreiche punktuelle Boden- und Grundwasserbelastungen mit Arsen und Blei festgestellt. Die Kontaminationsgehalte im Boden variieren von $100 \mathrm{mg} / \mathrm{kg}$ TS bis $27000 \mathrm{mg} / \mathrm{kg}$ TS, im Grundwasser liegen punktuelle Belastungen bis $100.000 \mathrm{mg} / \mathrm{l}$ vor.

Aufgrund der Standortbesonderheiten:

- inhomogene Schadstoffverteilung,

- bebauter Standort (zahlreiche Gebäude mit hohem Publikumsverkehr, ober- und unterirdische Versorgungsleitungen) sowie

- sehr hohe punktuelle Arsen- und Bleibelastungen sind konventionelle Bodensanierungs- bzw. -sicherungsverfahren nicht geeignet. Die elektrokinetische Sanierung, die bereits vor einigen Jahren an der TFH Wildau in Laborversuchen erprobt wurde, ist für solche Standorte gut geeignet und in manchen Fällen die einzig mögliche Sanierungsvariante.

Über die Problematik des Systems Arsen-Boden, arsenkontaminierte Böden und Möglichkeiten der Sanierung solcher Böden an bebauten Standorten lagen keine zusammenfassenden Erkenntnisse vor.

\subsection{Bewertung des Gefährdungspotenzials von Arsen}

Im Rahmen des Forschungsvorhabens des Bundesministeriums für Bildung und Forschung (BMBF) „Erarbeitung eines Leitfadens für die Anwendung elektroinduzierter in-situ-Sanierungsverfahren für bebaute Altlastenstandorte" (FK 14911103) wurde eine Literaturstudie zum Thema „Erfahrungen und Ergebnisse bei der Gefahrenbewertung sowie der Sanierung arsenkontaminierter Standorte im In- und Ausland" durchgeführt [2]. Diese Studie erfasst die Ergebnisse der Auswertung verschiedener Datenbanken und zahlreicher Umweltliteratur.

Für die Bewertung des Gefährdungspotenzials von Arsen und die erfolgreiche Sanierung arsenkontaminierter Altlasten ist es notwendig, Arsen-Spezies zu identifizieren und zu quantifizieren und deren Bindung an die Bodenbestandteile zu klären. Diese Aufgabe ist nur komplex zu lösen, weil Arsen in mehreren Oxidationsstufen vorkommt. Art und Menge der dabei entstehenden Arsen-Spezies, deren Mobilität und Toxizität sind sehr stark von äußeren Faktoren wie dem pH-Wert, RedoxBedingungen u. v. a. abhängig. Mit nur einem Analyseverfahren ist es beim heutigen Stand der Analytik nicht möglich, gesicherte Aussagen über die Mengen und Art von Arsen-Spezies, die gleichzeitig in einem FeststoffFlüssigkeit-System wie im Boden vorkommen, zu treffen. Über die Ergebnisse unserer Untersuchungen wird in Teil 2 dieser Reihe berichtet.

\subsection{Das Element Arsen in der Umwelt, natürliche Arsenvorkommen, Arsen- quellen anthropogenen Ursprungs}

Die wichtigsten Ergebnisse der durchgefuihrten Recherchen sind im Folgenden in kurzer Form dargestellt.

Das Element Arsen ist ein natürlicher Bestandteil der Erdkruste. Mit dem Massenanteil von ca. 5 x 10-4\% (entsprechend $5 \mathrm{~g}$ pro Tonne Gestein) ist es relativ selten (Arsen steht in der Häufigkeit der Elemente an 20. Stelle). In geringen Konzentrationen ist Arsen in allen Böden, Gesteinen und Pflanzen nachweisbar. Einen Überblick über die wichtigsten arsenhaltigen Gesteine, Mineralien und Böden ist in [3-10] gegeben. Insgesamt sind mehr als 2000 arsenhaltige Mineralien identifiziert. Die meistverbreiteten arsenhaltigen Mineralien sind Metallarsenide, Arsensulfide und arsenhaltige Fahlerze. Das Arsen ist in vielen sulfidischen Erzen als Begleitelement vorhanden, so dass daraus gewonnene Metalle meist 
Arsen enthalten. Als Verwitterungsprodukt von Arsenerzen findet man manchmal auch das Arsentrioxid $\mathrm{As}_{2} \mathrm{O}_{3}$. Man findet das Arsen auch im Schwefel der Vulkane, in Steinkohle und im Meerwasser.

Böden, die durch landwirtschaftliche oder industrielle Nutzung nicht kontaminiert sind, enthalten von 0,1 bis $40 \mathrm{mg} / \mathrm{kg}$ TS Arsen (Durchschnittwert ca. $5 \mathrm{mg} / \mathrm{kg}$ TS). Da die Böden durch die Verwitterung der Gesteine entstehen, entspricht die Arsenkonzentration im Boden dem Arsengehalt im entsprechenden Gestein. Arsenanreicherungen findet man insbesondere in Böden über sulfidischen Gesteinen und in Böden, die intensiv mit Pestiziden bearbeitet wurden.

In der Natur liegt das Arsen meistens in den Oxidationsstufen + III und +V vor. Als gediegener Arsen kommt es gelegentlich als Scherbenkobalt vor.

Es sind zwei kristalline enantiotrope Modifikationen von Arsenoxiden (Arsen in der Oxidationsstufe + III) und eine amorphe Modifikation bekannt:

\section{Kristalline enantiotrope Modifikationen}

a) Die kubische Modifikation Arsenolith $\mathrm{As}_{2} \mathrm{O}_{3}$ (Arsenit, Arsenbluite)

Die Kristallform ist oktaedrisch und ein Verwitterungsprodukt von Arsenmineralien, vor allem von Arsenkies, gediegenem Arsen, Enargit, Tennantit und Speiskobalt. Es kommt zusammen mit Claudetit, Kobaltbliite, Realgar und Auripigment vor.

Eine juvenile Bildung findet bei Gruben- und Flözbränden statt.

b) Die monokline Modifikation Claudetit $\mathrm{As}_{2} \mathrm{O}_{3}$ (Rhomarsenit, Arsenphyllit)

Die Kristallform ist prismatisch, dünntafelig und wird sekundär durch die Oxidation von Arsenmineralien zusammen mit Arsenolith, Realgar, Auripigment oder gediegenem Schwefel gebildet. Häufig wurde dieser auch als Sublimationsprodukt bei Grubenbränden gefunden.

Die Umwandlungstemperatur beider Modifikationen liegt bei ca. $-13^{\circ} \mathrm{C}$, die kubische Modifikation Arsenolith ist stabil bei tiefen Temperaturen, die monokline Modifikation Claudetit bei höheren Temperaturen. Die Umwandlungsgeschwindigkeit ist außerordentlich gering, so dass die beiden Modifikationen bis zu ihren Schmelztemperaturen nebeneinander existieren können.

Amorphe Form von $\mathrm{As}_{2} \mathrm{O}_{3}$

Durch Abkühlen von $\mathrm{As}_{2} \mathrm{O}_{3}$-Schmelzen, Kondensation von $\mathrm{As}_{2} \mathrm{O}_{3}$-Dämpfen oder Sublimation von einigen arsenhaltigen kristallinen Verbindungen erhält man die amorphe Modifikation von $\mathrm{As}_{2} \mathrm{O}_{3}$.

Die Löslichkeit von kristallinen Formen im Wasser ist gering, dagegen löst sich die amorphe Form des Arsentrioxids viel mehr im Wasser.

Die Verwitterungsprozesse, vor allem die Erosionsvorgänge, führen zu einer Arsenmobilisierung und zum Übergang der wasserlöslichen Arsenverbindungen in die wässrige Phase.
Die Primärprodukte der Verwitterung von Arsenmineralien sind:

- bei oxidierenden Bedingungen Arsenate - Salze von Arsensäure $\mathrm{H}_{3} \mathrm{AsO}_{4}$

- bei reduzierenden Bedingungen Arsenite - Salze von arsenigen Säuren

Die arsenige Säure $\mathrm{H}_{3} \mathrm{AsO}_{3}$ bzw. $\mathrm{As}(\mathrm{OH})_{3}$ ist amphoter. Die arsenige Säure kann in Abhängigkeit von Reaktionspartnern oxidierend oder reduzierend wirken. Diese Säure ist im freien Zustand nicht bekannt. Der isoelektrische Punkt der arsenigen Säure liegt bei einem pHWert von ca. 4.

\subsection{Arsen und seine Wechselwirkung mit Bodenbestandteilen, Einfluss der Boden- zusammensetzung auf das Verhalten von Arsen}

\section{Problemstellung}

Es ist bekannt, dass die Bodenzusammensetzung einen entscheidenden Einfluss auf das Verhalten von Arsen im Boden hat. Diese Tatsache erschwert sehr stark die Möglichkeit, allgemein gültige Aussagen über die Oxidationsstufe, Bindungsform und Mobilität von Arsen zu treffen. Diese Aussagen gelten jeweils für einen Bodentyp bestimmter chemischer und mineralogischer Zusammensetzung.

\section{Zusammensetzung und der pH-Wert des Bodens}

Die Zusammensetzung und der pH-Wert des Bodens bestimmen in erster Linie, mit welchen Kationen und in welchem Maße das Arsenation $\left[\mathrm{AsO}_{4}\right]^{3-}$ eine Verbindung eingeht. Die Phasenzusammensetzung arsenhaltiger Bodenkomponenten ändert sich unter dem Einfluss der äußeren Bedingungen, besonders durch Änderung des pH-Wertes und des Redoxpotenzials. Zum Beispiel liegt das Arsen in durchluifteten Böden im pH-Bereich von 4 bis 8 meistens gebunden an o. g. Oxide und Hydroxide als Arsenat-Anion vor. Durch die Reduktion von Fe(III) zu $\mathrm{Fe}(\mathrm{II})$ und von $\mathrm{Mn}$ (III) bzw. Mn(IV) zu Mn(II) können unlösliche Hydroxide und Oxide in lösliche Verbindungen überführt werden. Dadurch verschieben sich die Verhältnisse zwischen den einzelnen Phasen.

\section{Adsorptionsprozesse}

Die Adsorption von Arsenspezies an verschiedenen Bodenbestandteilen sind sehr vielfältig und wirkungsvoll. Diese Phänomene werden technisch genutzt, z. B. wird die Adsorption an hydratisierten Metalloxidoberflächen, insbesondere an Eisen(III)hydroxiden zur Arsenentfernung in der Wasseraufbereitung eingesetzt [11, 12].

Einige andere Bodenbestandteile wie Tonmineralien mit verschiedenen Schichtfolgen aus $\mathrm{SiO}_{2}$-Tetraeder und $\mathrm{Al}_{2} \mathrm{O}_{3}$-Oktaeder und dazwischen gelagerten unterschiedlichen Kationen und die Huminstoffe beeinflussen auch die Adsorption und damit die Mobilität von Arsenspezies im Boden. Die Affinität von Arsenverbindungen an Tonmineralien hängt von der Zusammensetzung und der Struktur der Tonmineralien ab. Bezüglich der Wechselwirkung von Arsenverbindungen mit Tonmineralien sind 
kaum gesicherte Erkenntnisse aus der Literatur bekannt. Es sind lediglich einige wenige Hinweise auf unterschiedliches Adsorptionsverhalten von Schwermetallen an Tonmineralien vorhanden [13-16]. Die Adsorptionsprozesse an Metalloxid- und Tonmineralienoberflächen bestimmen und kontrollieren die Arsenkonzentration in Grundwasserleitern. Noch komplizierter sind diese Zusammenhänge unter dem Einfluss elektrischer Felder, wie es bei der elektrokinetischen Bodensanierung der Fall ist. Die sandigen Böden mit wenig Tonmineralien, Eisen-, Manganoxiden und -hydroxiden und wenig organischem Material wie Huminstoffe können nur geringe Mengen Arsenspezies durch Adsorption binden [17, 18].

Obwohl in sandigen Böden die Menge von Huminstoffen verhältnismäßig gering ist, bewirken diese Bodenbestandteile einen weiteren, die Analytik erschwerenden Einfluss. Sie besitzen polare und unpolare Bereiche sowie eine Vielzahl funktioneller Gruppen, wie z. B. Carbonyl-, Carboxyl-, Keto- und OH-Gruppen. Diese Stoffe reagieren ampholytisch, in gelöstem Zustand besitzen sie elektrolytische Eigenschaften und sind oberflächenaktiv. Huminstoffe können durch ihre Wechselwirkung mit Metallen und Tonmineralien (z. B. über die Bildung von Komplexen) entscheidend die Mobilität von Schadstoffen im Boden verändern und damit die Ergebnisse der elektrokinetischen Bodensanierung beeinflussen. Die Wirkungsmechanismen der Huminstoffe im Boden und die Art der Bindung der Arsen- und Schwermetallverbindungen an diese sind noch nicht endgültig geklärt. Die Identifikation und Quantifizierung von verschiedenen Arsenspezies in Böden wird weiterhin durch im Boden enthaltene Lösungen und Gasen erschwert.

\section{Mikroorganismen}

Die Mikroorganismen, wie Bakterien und Schimmelpilze, und ihre Stoffwechselprodukte können Arsenat zu Arsenit reduzieren und das Arsenit zu Methylarsonsäure methylieren und in weiteren Zyklen durch Oxidationsbzw. Reduktionsvorgänge zum Entstehen von verschiedenen methylierten Arsenverbindungen führen. Eine Bodenprobe kann folgende Arsenverbindungen enthalten: Arsenate, Arsenite, Methylarsonsäure $\mathrm{CH}_{3} \mathrm{AsO}_{3} \mathrm{H}_{2}$ und Dimethylarsinsäure $\left(\mathrm{CH}_{3}\right)_{2} \mathrm{AsO}(\mathrm{OH})$ [4].

Zur Beurteilung des As-Gefährdungspotenzials fuir Pflanzen wegen der starken Interaktion von Arsenat mit Phosphat kann das Gefährdungspotenzial von Arsenkonzentrationen im Boden nur bei einer gleichzeitigen Beurteilung des Phosphatzustandes der Böden erfasst werden. Arsen (im Unterschied zu Schwermetallen) wird nur von wenigen Pilzarten aufgenommen und über die Konzentration im Boden hinaus gespeichert. Es ist z. B. von wild wachsenden Agaricus-Arten (Champignons) bekannt, dass sie Arsen akkumulieren können. Die Bestimmung des As-Gehaltes in wildwachsenden Pflanzen kann nicht als Biomonitoring dienen.

\section{Anorganisches Arsen}

Die Untersuchungen über das Verhalten von anorganischem Arsen in Böden [4] unter Berücksichtigung der Anwesenheit von Al-, Cu-, Fe-, Zn-, Mn-, Cd-, Ca-, Ni- und
Pb-Verbindungen ergaben, dass Calciumarsenat $\mathrm{Ca}_{3}\left(\mathrm{AsO}_{4}\right)$ die stabilste Arsenatphase in aeroben, alkalischen Böden ist. Die Stabilität der tertiären Arsenate nimmt in der folgender Reihenfolge ab:

$\mathrm{Ca}>\mathrm{Mn}(\mathrm{II})>\mathrm{Cd}>\mathrm{Pb}>\mathrm{Cu}(\mathrm{II})>\mathrm{Al}>\mathrm{Fe}(\mathrm{III})>\mathrm{Zn}>\mathrm{Ni}$ In sauren Böden ist der Calciumarsenat dagegen instabil und der Manganarsenat bleibt stabil. In reduzierenden sauren Böden sind auch Arsensulfide und Arsenite stabil.

Arsenit (III) und Arsenat (IV) in wässrigen Lösungen, Lösungsverhalten von As in anthropogen belasteten Böden

Die Arsenspezies Arsenat (III) und Arsenat (IV) sind anionische Verbindungen, die sich von den dreiprotonigen Säuren - Arseniger Säure $\mathrm{H}_{3} \mathrm{AsO}_{3}$ bzw. Arsensäure $\mathrm{H}_{3} \mathrm{AsO}_{4}$ - ableiten. Durch entsprechende Änderungen des Redoxmilieus lassen sich diese Spezies ineinander uiberfuihren:

Redox-Paar As-(V) / As-(III)

$\mathrm{H}_{3} \mathrm{AsO}_{3}+\mathrm{H}_{2} \mathrm{O} \Leftrightarrow \mathrm{H}_{3} \mathrm{AsO}_{4}+2 \mathrm{H}^{+}+2 \mathrm{e}^{-} \quad \mathrm{E}_{\mathrm{o}}=+0,56 \mathrm{~V}$

Damit diese Reaktion ablaufen kann, muss ein Reaktionspartner zur Verfuigung stehen, der diese Elektronen aufnimmt. Während Arsenat (III) durch diese Reaktion zu Arsenat (IV) oxidiert wird, wird der Reaktionspartner reduziert. Alle Redox-Reaktionen sind pH-abhängig. Zur Oxidation von Arsenat (III) sind somit a priori alle Redoxpaare geeignet, die ein größeres Normalpotenzial aufweisen als 0,56 V des Redox-Paares As-(V)/As-(III).

An solchen arsenbelasteten Standorten wie Wildau sind im Boden zahlreiche eisenhaltige Verbindungen enthalten, die u. a. als Verwitterungsprodukte der Erze auftreten. Eisen(III)hydroxid besitzt eine reaktive Oberfläche, die in Abhängigkeit vom pH-Wert sowohl Anionen als auch Kationen unspezifisch adsorbieren kann. Eine Eisen(III)hydroxid-Oberfläche verhält sich in wässriger Lösung amphoter. Die Hydroxyl-Gruppen an der Feststoffoberfläche können sowohl ein Proton abgeben (Negativierung der Oberfläche) als auch aufnehmen (Positivierung der Oberfläche). Der pH-Wert der geringsten Eisen(III)hydroxid-Löslichkeit entspricht dem $\mathrm{pH}$ Wert, bei dem die Nettooberflächenladung gleich Null ist. Für $\mathrm{Fe}(\mathrm{OH})_{3}-$ Goethit - ist es bei dem pH-Wert $=$ 7,8 der Fall. Bei pH-Werten darunter sind die Oberflächen positiv [geladen], bei pH-Werten darüber negativ [geladen]. Eisenverbindungen erschweren im Allgemeinen die Mobilität des Arsens im Boden.

\subsection{Schlussbemerkung}

Die Bodenzusammensetzung hat einen entscheidenden Einfluss auf das Verhalten von Arsen im Boden. Diese Tatsache erschwert sehr stark die Möglichkeit, allgemein guiltige Aussagen über die Oxidationsstufe, Bindungsform und Mobilität von Arsen zu treffen. Diese Aussagen gelten jeweils furr einen Bodentyp bestimmter chemischer und mineralogischer Zusammensetzung. Die Sanierung arsenkontaminierter Standorte stellt ein gro- 
ßes Problem dar. Einzelne Standorte sind schlecht bzw. gar nicht miteinander vergleichbar, da das Arsenverhalten im Boden sehr stark von der Form der Arsenbindung, der Bodenart und den Umgebungsbedingungen abhängt. Durch die Überlagerung der Schadstoffe infolge im Laufe der Zeit unterschiedlicher Nutzungen der Standorte und unterschiedlicher Bodenparameter an unterschiedlichen Standorten wird jede Verdachtsfläche und jeder Standort zu einem Einzelfall.

\section{Teil 2: Untersuchungen an arsen- kontaminierten Böden}

\section{Udo Hellwig (ITVA), Olga Böhme, Werner Schmitz}

\subsection{Auswahl der Untersuchungsmethoden}

Im Rahmen des BMBF-Forschungsvorhabens „Erarbeitung eines Leitfadens für die Anwendung elektroinduzierter in-situ-Sanierungsverfahren für bebaute Altlastenstandorte“ (FK 14911103) [19] orientiert an die Lokalität Gelände TFH Wildau wurden zahlreiche aufeinander abgestimmte Bodenuntersuchungen durchgeführt, um Kenntnisse über die mineralogische und chemische Bodenzusammensetzung und Gefuigeausbildung, die physikalischen und geophysikalischem Bodeneigenschaften, die chemischen und physikalischen Eigenschaften und das Verhalten von Kontaminanten zu gewinnen.

Die Auswahl geeigneter analytischer Untersuchungsverfahren hat unter besonderer Berücksichtigung der Möglichkeiten und Grenzen einzelner Methoden, der Nachweisgrenzen, der Reproduzierbarkeit, der Empfindlichkeit gegenüber dem Umgebungsmilieu und der Umweltbedingungen, der Anwendbarkeit auf kontaminierte Böden (auch bei Anwesenheit anderer Kontaminanten) und der Praktikabilität stattgefunden.

Die vollständige Charakterisierung von Bodenmaterial auf Arsengehalt ist sehr komplex und kann nach dem heutigem Stand des Wissens nur mehrstufig erfolgen. Wichtigste Schritte dabei sind:

1) Bestimmung des Gesamtarsengehaltes,

2) Identifizierung und quantitative Bestimmung von Arsenverbindungen,

3) Bestimmung, an welche Bodenbestandteile diese Arsenverbindungen gebunden sind.

\section{zu 1) Bestimmung des Gesamtarsengehaltes}

Eine Angabe der Gesamtarsenkonzentration als Grad der Arsenbelastung ist die verbreitetste Variante, um den Arsenschaden zu charakterisieren, sie gibt aber eine nur unzureichende Information über die Risiken für den Menschen und den tatsächlichen Handlungsbedarf. Bei gleicher Gesamtarsenkonzentration könnte das Arsen als z. B. unlösliches bzw. schwerlösliches Eisenarsenat $\mathrm{FeAsO}_{4}$, als Arsensulfid $\mathrm{As}_{4} \mathrm{~S}_{4}$ bzw. $\mathrm{As}_{2} \mathrm{~S}_{2}$ oder als leichtlösliche Arsenverbindungen wie Alkaliarsenite, z. B. Natriumarsenit $\mathrm{NaH}_{2} \mathrm{AsO}_{3}$ und Kaliumarsenit $\mathrm{KH}_{2} \mathrm{AsO}_{3}$, vorkommen (Erdalkaliarsenite sind dagegen schwerlös- lich). Die Bestimmung von Totalarsen ist mit verschiedenen Methoden möglich, und man erhält dabei gesicherte Erkenntnisse [3, 4, 20, 21]. Bei unseren Untersuchungen wurden spektroskopische Methoden und klassische chemische Analysen angewandt.

zu 2) Identifizierung und quantitative Bestimmung von Arsenverbindungen

Die Identifizierung und die quantitative Bestimmung einzelner Arsenverbindungen in kontaminierten Böden bereitet sehr große Schwierigkeiten. In der Literatur gibt es aus diesem Grund wenig gesicherte Erkenntnisse dazu. Die Ursachen dafür sind sehr vielfältig:

- Zum einen liegen die Arsenverbindungen in Böden meistens nicht als unlösliche reine Salze der im Boden vorhandenen Kationen vor, sondern bilden verschiedene Assoziate mit Fe(III)-, Al(III)-, Mn(III)- und $\mathrm{Mn}(\mathrm{V})$-Hydroxiden und -Oxiden und Tonmineralien.

- Zum anderen ist die absolute Arsenmenge, die an eine einzelne mineralogische Phase gebunden werden kann, in der Regel so gering, dass diese Phase unter bzw. an der Nachweisgrenze der für Phasenbestimmungen gebräuchlichen Analyseverfahren, wie z. B. der Röntgendiffraktometrie, liegt.

Die Elektronenstrahlmikroanalyse wurde bereits für die Identifizierung von Schwermetallverbindungen im Boden eingesetzt [22]. Für As-Spezies sind solche Untersuchungen nicht bekannt.

Die Phasenzusammensetzung arsenhaltiger Bodenkomponenten ändert sich unter dem Einfluss der äußeren Bedingungen, besonders durch die Änderung des pHWertes und des Redoxpotenzials. Zum Beispiel liegt das Arsen in durchluifteten Böden im pH-Bereich von 4 bis 8 meistens gebunden an o.g. Oxide und Hydroxide als Arsenat-Anion vor. Durch Reduktion von Fe(III) zu Fe(II) und von Mn(III) bzw. Mn(IV) zu Mn(II) können unlösliche Hydroxide und Oxide in lösliche Verbindungen überfuihrt werden. Dadurch verschieben sich die Verhältnisse zwischen einzelnen Phasen.

zu 3) Bestimmung, an welche Bodenbestandteile diese Arsenverbindungen gebunden sind

Die Kenntnis, an welche Bodenphasen das Arsen gebunden ist, hat große Bedeutung für die ökologische Wirkung des Arsens. In den letzten Jahren wurden deshalb verschiedene sequenzielle Extraktionsverfahren entwickelt, die es ermöglichen, das Gesamtarsen in charakteristische Arsenfraktionen aufzuteilen, die an bestimmte Bodenkomponenten gebunden sind. Eine umfassende Übersicht der sequenziellen Extraktionsmethoden für viele Elemente für Sedimentgesteine ist in [23] zu finden. In den so erhaltenen Extrakten wird meist nur Totalarsen bestimmt.

Es gibt bis heute keine standardisierte Methode der sequenziellen Extraktion, auch für die Arsenbestimmung sind mehrere Varianten möglich. In vielen Arbeitsgruppen sind eigene Verfahren mit unterschiedlichen Extraktionsmitteln und unterschiedlicher Anzahl an Schritten entwickelt worden. Im Rahmen des Forschungsvorhabens wurden auch Verfahren zu sequenzieller ArsenExtraktion speziell aus den Wildauer Bodenproben ent- 
wickelt [1]. Um die einzelnen Arsenverbindungen zu identifizieren, reicht diese Methode aber nicht. Ursache dafuir sind die Adsorptionsprozesse von Arsenspezies an verschiedenen Bodenbestandteilen, die sehr vielfältig und wirkungsvoll sind.

Analytische Methoden zur Beurteilung des Arsen-Gefährdungspotenzials und zur Identifizierung und Quantifizierung von Arsenverbindungen unterscheiden sich in Abhängigkeit davon, ob Arsen anorganisch oder organisch vorliegt. In den arsenkontaminierten Wildauer Böden liegt Arsen, bedingt durch die Entstehungsgeschichte, in anorganisch gebundener Form vor. Diese Tatsache bestimmte die Auswahl der eingesetzten Analyseverfahren. Begleitend dazu wurden kontinuierlich die Messungen des Grundwasserspiegels (bei ca. 2,5 $\mathrm{m})$, der elektrischen Leitfähigkeit und des pH-Werts des Grundwassers durchgeführt.

Standortbezogene Analysemethoden (Untersuchungen zur Elution und Mobilisierbarkeit von Schwermetallen, sequenzielle Extraktion) ergaben, dass das Wasser weder Blei noch Arsen in nennenswertem Maße eluiert. Salzsäure eluiert zunächst beide Elemente, die Eluierbarkeit von Arsen nimmt aber mit Zunahme der Bleikonzentration und der Anzahl der Durchläufe ab. Es wurde festgestellt, dass Blei die Eluierbarkeit von Arsen behindert [19].

\subsection{Röntgenographische Charakterisierung von Bodenproben aus Wildau}

\section{Zu allgemeinen Grundlagen der Röntgendiffraktometrie}

Die Röntgenstrukturanalyse ist ein Verfahren zur Bestimmung der Atomanordnung im Kristallgitter durch Röntgenstrahlen. Die Wellenlänge der Röntgenstrahlen liegt in der Größenordnung der Atomabstände im Kristallgitter $\left(10^{-10} \mathrm{~m}\right)$, deshalb dient das Kristallgitter hierbei als räumliches Beugungsgitter und erzeugt Strahlungsinterferenzen. Die Interferenzmaxima werden immer dann beobachtet, wenn die Bedingung

$$
\mathrm{n} \lambda=2 \mathrm{~d} \sin \theta \quad \text { (Braggsche Gleichung) }
$$

erfuillt wird und der Gangunterschied einfallender Wellen $\Delta=\mathrm{n} \lambda$ ein ganzzahliges Vielfaches der Wellenlänge ist $(n=1,2,3 \ldots)$. Dabei sind $\lambda$ - Wellenlänge der Röntgenstrahlen, $\theta$ - Einfallwinkel des Röntgenstrahlenbündels und d - Netzebenenabstand im Kristallgitter.

Die Braggsche Gleichung zeigt, dass bei der vorgegebenen Wellenlänge nur in ganz bestimmten Raumrichtungen die Interferenzmaxima der gebeugten Röntgenstrahlen auftreten, die scharf ausgebildet sind, da an der Beugung eine Vielzahl von Netzebenen beteiligt ist.

Aus der Lage, Anzahl und Intensität der Interferenzmaxima (Reflexe) wird die Struktur des beugenden Kristalls bestimmt. Die Interferenzmaxima sind zu erwarten bei folgenden apparativen Bedingungen: a) bei festgehaltenen q muss I variabel sein (Einkristallverfahren - Laue-Methode)

b) bei festgehaltener I (monochromatische Röntgenstrahlung) muss q variabel sein.

Die Variation von q kann entweder durch Drehen des Kristalls erreicht werden (Drehkristallverfahren) oder dadurch, dass eine große Zahl kleiner Kristalle in beliebiger Orientierung (Kristallpulver) durchstrahlt wird (Debye-Scherrer-Verfahren). Die Registrierung bzw. Wiedergabe der Interferenzen ist in Form von Röntgenbildern auf Filmen und Platten oder als Röntgendiffraktogramm möglich.

Die im Folgenden beschriebenen Bodenuntersuchungen sind nach dem Bragg-Brentano-Verfahren durchgefuihrt.

\section{Untersuchte Proben}

a) Baggerschurf aus verschiedenen Tiefen (oberflächennahe Proben, Tiefen 0,6 und 1,5 m), dazu Vergleichsprobe Sand Niederlehme (Proben gleicher geologischer Herkunft wie Boden Wildau)

b) Proben aus der Brunnenbohrungen

Die röntgenographische Untersuchung von sandhaltigen Proben stellt ein großes Problem dar. Es ist allgemein bekannt, dass der Quarz aufgrund seiner kristallographischen Eigenschaften sogar bei Mengen kleiner 1 Mass. $\%$ sehr starke und zahlreiche Reflexe liefert, die die gesicherte Identifizierung von anderen, in der Probe anwesenden kristallinen Phasen entscheidend erschweren können. In unseren Untersuchungen wurden speziellen Präparationsmethoden angewandt.

\section{Aufnahmebedingungen}

- Röntgendiffraktometer Kristalloflex D 5000, Fa. Siemens

- Anregungsspannung 45 kV, Leistung der Röntgenröhre $1575 \mathrm{~W}$

- Strahlung Cu $\mathrm{k}_{\mathrm{a}}$

- Winkelbereich 2q $8-80^{0}$

- ortsempfindlicher Detektor (OED) ohne Monochromator

Alle identifizierten bzw. in Frage kommenden Phasen wurden aufgrund ihrer Raumgruppe auf eine mögliche Texturausbildung überprüft.

Ein Vergleich der Übersichtsaufnahmen von allen untersuchten Proben ergab, dass in allen Proben der Quarz erwartungsgemäß die vorherrschende Phase ist. Außer Quarz wurden Hämatit, Montmorillonit, Kaolinit und Gips nachgewiesen. Die Identifizierung der Phase Hämatit bedeutet hier wie auch in anderen eisenhaltigen Bodenproben den Nachweis der Anwesenheit von Eisenoxiden und -hydroxiden ohne Hinweise auf die genaue chemische Zusammensetzung dieser Phasen. Diese eisenhaltigen Phasen sind teilweise amorph oder teilkristallin und liefern im Röntgendiffraktogramm ähnliche Reflexe wie Hämatit. Eine genaue Unterscheidung dieser Eisenoxide und -hydroxide ist nur in gut ausgebildeten kristallinen Phasen möglich, was auf Sedimentgesteine bzw. Fällungs- und Umwandlungsprodukte unter Umwelteinflüssen kaum zutrifft. 
Die Auswertung der Diffraktogramme ermöglichte, wichtige Aussagen über die sehr komplizierte mineralogische Phasenzusammensetzung der untersuchten kontaminierten Bodenproben aus Wildau zu machen. Es sind folgende, kontaminantenhaltige Phasen nachgewiesen:

- Bleioxidsulfat $\mathrm{Pb}_{5} \mathrm{O}_{4} \mathrm{SO}_{4}$. (Im Stoffsystem $\mathrm{PbO}-\mathrm{PbSO}_{4}$ sind mehrere kristalline und amorphe Phasen bekannt, die stabilste von allen ist Bleioxidsulfat $\mathrm{Pb}_{5} \mathrm{O}_{4} \mathrm{SO}_{4}$. Die Anwesenheit anderer Phasen aus diesem System ist zu vermuten.) Diese Phase wurde eindeutig in mehreren Proben röntgenographisch nachgewiesen.

- Gabrielsonit $\mathrm{PbFeAsO}_{4}(\mathrm{OH})$ (enthält gleichzeitig beide Kontaminanten $\mathrm{Pb}$ und $\mathrm{As}, \mathrm{Pb}$ als Kation, $\mathrm{As}(\mathrm{V})$ als Anion $\left[\mathrm{AsO}_{4}\right]^{3-}$ (Beispiel Abb. 1)). Dieses Mineral ist sehr selten, wurde bis jetzt nur einmal 1967 in Schweden gefunden und beschrieben (JCPDS 1998, Nr.200583).

- Arsen in der Oxidationsstufe (III) ist als Mineral Claudetit $\mathrm{As}_{2} \mathrm{O}_{3}$ und auch als Mineral Arsenolith $\mathrm{As}_{2} \mathrm{O}_{3}$ vorhanden.

In den Wildauer Bodenproben sind mit Sicherheit viel mehr kristalline bzw. amorphe blei- und arsenhaltige Phasen vorhanden. Deren sicherer Nachweis scheitert aber an den Nachweisgrenzen der verwendeten Methoden. Zum Beispiel kann die Nicht- oder Anwesenheit von Arsentrioxid in amorpher Form, die bekanntlich leichter wasserlöslich als kristalline Formen ist, mit der Röntgendiffraktometrie nicht nachgewiesen werden.

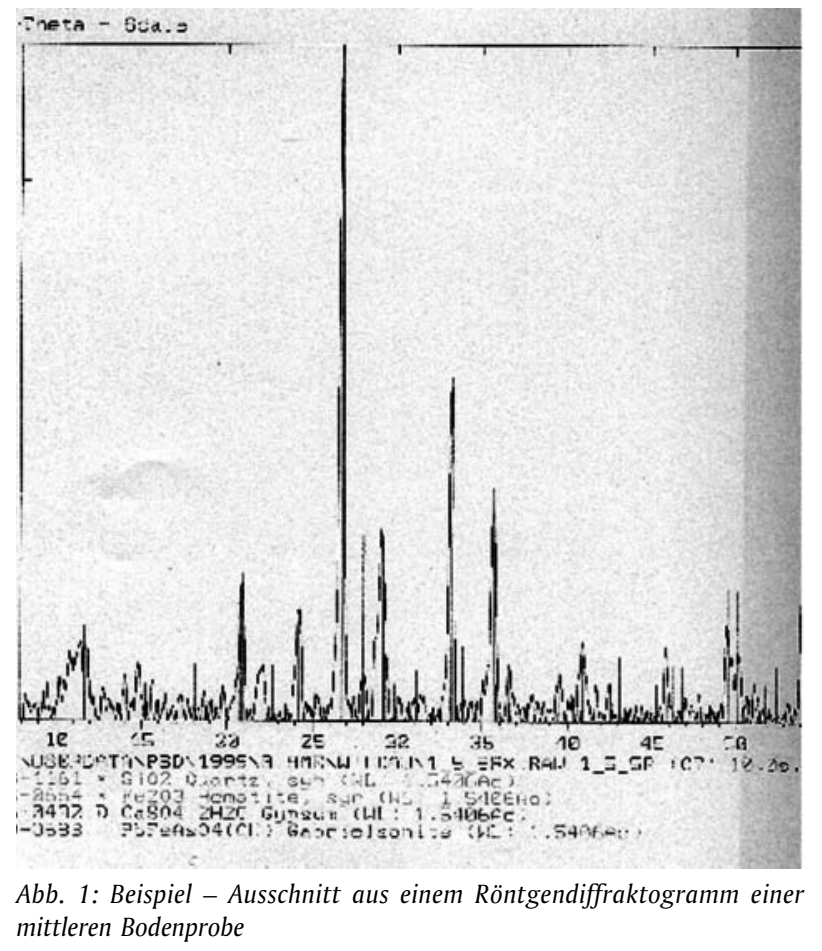

\subsection{Lichtmikroskopische, rasterelektronenmi- kroskopische und ESMA-Untersuchungen}

Lichtmikroskopische Untersuchungen ergaben, dass die Bodenproben sehr inhomogen aufgebaut sind: Neben Sandkörnern treten unterschiedlich gefärbte Bestandtei- le auf, die in der Kornform und -größe sehr auffällig sind. In allen Proben sind verschiedene Arten von Kohlepartikeln und unterschiedliche weißliche, gelbliche und glimmerartige Partikel vorhanden. Der Vergleich der lichtmikroskopischen Aufnahmen von kontaminierten Proben mit unterschiedlichem Arsengehalt ergab, dass es keine signifikanten Unterschiede zwischen einzelnen Proben in Abhängigkeit vom Kontaminantengehalt gibt.

\section{Rasterelektronenmikroskopische und ESMA-Untersu- chungen (WDS- und EDS-System) von Bodenproben aus Wildau}

Zum Prinzip: Wechselwirkungen von Elektronenstrahlen im Rasterelektronenmikroskop (REM) mit der Probe fuihren zur Entstehung zahlreicher Signale, die fuir analytische Zwecke genutzt werden können. Einige der wichtigsten im REM nutzbaren Signale sind: rückgestreute Elektronen, Sekundärelektronen und Röntgenstrahlen. Die Untersuchung mit dem REM erlaubt im Vergleich zur Lichtmikroskopie wesentlich höhere Vergrößerungen bei größeren Schärfentiefen, z. B. bei 1000 facher Vergrößerung beträgt die Schärfentiefe im Lichtmikroskop 1 $\mathrm{mm}$, im Rasterelektronenmikroskop ca. $40 \mathrm{~mm}$. Durch Kombination von REM mit verschiedenen Signaldetektoren ist es möglich, die Oberflächenstrukturen und -rauhigkeit abzubilden sowie die stoffliche Zusammensetzung der Proben und die Verteilung einzelner Elemente innerhalb der Probe zu bestimmen. Für die Abbildung von Oberflächenstrukturen werden am meisten Sekundärelektronen und rückgestreute Elektronen genutzt, Elementverteilungsbilder werden mit Hilfe von Sekundärelektronen gemacht, der Nachweis von Röntgenstrahlen - Elektronenstrahlmikroanalyse (ESMA, Mikrosonde) - dient der chemischen Analyse.

Der Nachweis von Röntgenstrahlen kann mit verschiedenen Detektoren erfolgen. Man unterscheidet dabei das WDS-System (Wellendispersive Spektrometer) (EDX) - und das EDS-System (Energiedispersive Spektrometer) - (WDX). Beide Systeme haben ihre Vorteile und Nachteile, die bei der Anwendung berücksichtigt werden müssen.

Rasterelektronenmikroskopische und ESMA-Untersuchungen wurden an verschiedenen Proben mit beiden Systemen (WDS- und EDS-System) durchgefuihrt.

Beispiel EDS-System-Aufnahmebedingungen:

- Philips XL 20 - Rasterelektronenmikroskop

- Mikrosonde EDAX USA DX-4i

- W-Kathode

- Belüftung - Stickstoff, Kühlung - flüssiger Stickstoff

Das Probematerial stammt wie bei frn röntgenographischen Untersuchungen aus verschiedenen Stellen des Geländes und Tiefen. Probenpräparation - Au-Bedampfung.

\section{Ergebnisse}

In fast allen untersuchten Proben ist $\mathrm{As}$ und $\mathrm{Pb}$ mit beiden ESMA-Verfahren als Element nachweisbar (Beispiel Abb. 2). Mit Hilfe von Elementverteilungsbildern wurde die Verteilung von Arsen innerhalb der Boden- 
proben untersucht und die Anwesenheit von Arsen in fast allen untersuchten Proben nachgewiesen. Über die Art der Arsenverbindungen erlaubt diese Methode keine Aussagen.

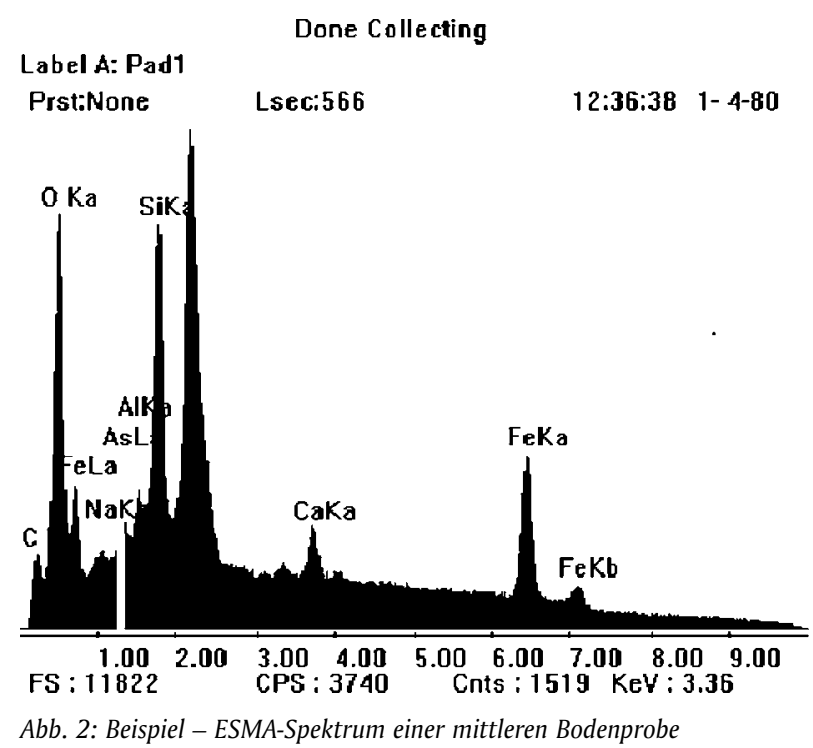

\subsection{Schlussbemerkung}

Der kontaminierte Boden aus Wildau weist folgende Besonderheiten auf:

- Die Gesamtkonzentration von $\mathrm{As}, \mathrm{Pb}$ und anderen Elementen ist nicht uiberall gleich und hat eine inhomogene Verteilung.

- Der Aufbau ist heterogen: mehrere Fraktionen, unterschiedliche Korngröße von feinkörnig (Tone) bis Brocken (Ziegelsteinreste, metallische Gegenstände), unterschiedliche chemische und mineralogische $\mathrm{Zu}$ sammensetzung, hoher Kontaminantengehalt.

- Der Grundwasserspiegel (bei ca. 2,5 m), die elektrische Leitfähigkeit und der pH-Wert des Grundwassers variieren in Abhängigkeit von der Jahreszeit und den Wetterverhältnissen.

- Der Anteil von organischem Material ist gering, Arsen und Blei sind anorganisch gebunden.

Der Unterschied im chemischen Verhalten und der Toxizität verschiedener Arsenverbindungen macht deutlich, wie wichtig es ist, die einzelne Spezies zu identifizieren und zu quantifizieren, statt nur den Gesamtgehalt an Arsen zu bestimmen. Die Adsorbierbarkeit von As(V) ist grundsätzlich besser als die von As(III) und nimmt mit höherem Eisengehalt des Bodens zu. Es wird tendenziell auch eine Zunahme der Adsorption mit steigendem pH-Wert beobachtet, die Untersuchungsergebnisse sind aber teilweise widersprïchlich.

Es gibt bis heute keine standardisierte Methode der sequenziellen Extraktion, auch fuir die Arsenbestimmung sind mehrere Varianten möglich. Die Aussagen zur Arsenmobilität nur aus den Ergebnissen der sequenziellen Extraktion abzuleiten, ist sehr vage. Um die einzelnen Arsenverbindungen zu identifizieren, reicht diese Methode nicht.
Die Identifikation und Quantifizierung von verschiedenen Arsenspezies in Böden und darin enthaltenden Lösungen und Gasen stellt ein großes, noch ungelöstes Problem dar.

Die gesicherten Aussagen über die Arsenverbindungen in kontaminierten Böden und über die physikalisch-chemische Bindung von Arsen an bestimmte Bodenbestandteile kann man nur durch eine kombinierte Anwendung von mehreren Methoden der Analytik gewinnen.

Es sind folgende, kontaminantenhaltige Phasen nachgewiesen:

- Bleioxidsulfat $\mathrm{Pb}_{5} \mathrm{O}_{4} \mathrm{SO}_{4}$

- Gabrielsonit $\mathrm{PbFeAsO}_{4}(\mathrm{OH})$ enthält gleichzeitig beide Kontaminanten $\mathrm{Pb}$ und $\mathrm{As}$ und ist ein seltenes Mineral (in der Literatur bis jetzt nur einmal beschrieben).

- Arsen in der Oxidationsstufe (III) ist als Mineral Claudetit $\mathrm{As}_{2} \mathrm{O}_{3}$ und auch als Mineral Arsenolith $\mathrm{As}_{2} \mathrm{O}_{3}$ vorhanden.

- $\mathrm{As}_{2} \mathrm{O}_{3}$ in amorpher Form ist mit großer Wahrscheinlichkeit in mehreren untersuchten Proben vorhanden. Dafür spricht die Tatsache, dass man in einigen Proben große Mengen von Arsen mit chemischen und spektroskopischen Methoden, die den Gesamtarsengehalt angeben, bestimmt hatte, aber mit den verwendeten röntgenographischen Methoden keine arsenhaltigen Phasen nachgewiesen werden konnten. Für den Nachweis von amorphen Phasen braucht man spezielle röntgenographische Verfahren, die im Rahmen des Forschungsvorhabens nicht zur Verfügung standen.

Die Tatsache, dass As(III) in verschiedenen Formen, auch amorph, im Boden auftritt, lässt eventuell die Widerspruiche bei der Bestimmung von mobilisierbaren und unmobilisierbaren Arsen-Anteilen nach der Methode der sequenziellen Extraktion im Boden erklären.

Die gewonnenen Ergebnisse bestätigen, dass die Sanierung von arsenkontaminierten, bebauten Standorten mit bekannten und verbreiteten Methoden wie Bodenwäsche nicht möglich ist. Eine Erfolg versprechende Alternative dazu ist die elektrokinetische Bodensanierung.

\section{Danksagung}

Für die Unterstuitzung bei der Probenpräparation und Anfertigung von Röntgenaufnahmen danken wir Herrn Dipl.-Ing. G. Kommichau (Universität Leipzig), bei der Durchfuihrung von rasterelektronenmikroskopischen und ESMA-Untersuchungen Herrn Prof. Dr. Rolle (TFH Wildau) sowie Herrn Dipl.-Ing. K. Wustig und Herrn Prof. Dr. J. Klose (FHTW Berlin), bei der Durchfuihrung von lichtmikroskopischen Untersuchungen Frau Prof. Dr. A. Richter (TFH Wildau) und Herrn Dipl.-Krist. R. Ries (TFH Wildau). 


\section{Teil 3: Elektrokinetische Boden- sanierung am Beispiel arsen- kontaminierter Böden Wildau}

\author{
Udo Hellwig (ITVA), Olga Böhme, Edgar Wetzig
}

\subsection{Einführung}

Aufgrund der o. g. Standortbesonderheiten wie die inhomogene Schadstoffverteilung, der bebaute Standort (zahlreiche Gebäude mit hohem Publikumsverkehr, oberund unterirdische Versorgungsleitungen) und die sehr hohen punktuellen Arsen- und Bleibelastungen im ungesättigten und gesättigten Bereich sind konventionelle Bodensanierungs- bzw. -sicherungsverfahren (z. B. Bodenwäsche, thermische Verfahren) nicht geeignet. Die durchgefuihrte Literaturrecherche ergab, dass über die Möglichkeiten der Sanierung solcher Böden an bebauten Standorten keine zusammenfassenden Erkenntnisse vorliegen. Im Rahmen des BMBF-Forschungsvorhabens „Erarbeitung eines Leitfadens fuir die Anwendung elektroinduzierter in-situ-Sanierungsverfahren für bebaute Altlastenstandorte“ (FK 14911103) wurde die Möglichkeit der Sanierung solcher Standorte mit elektrokinetischen Verfahren bewiesen.

\subsection{Vorteile der elektrokinetischen Verfahren für die Sanierung bebauter Standorte}

Die elektrokinetische Sanierung von Böden und Schlämmen ist ein innovatives Verfahren, mit dem in der gegenwärtigen Sanierungspraxis eine Lücke geschlossen wird. Diese Verfahren wurden bereits in Labor- und Technikumsversuchen eingesetzt [24-28].

Elektrokinetische Bodensicherungs- und -sanierungssysteme bieten folgende Vorteile:

- Durch die elektrische Barriere wird ein fortgesetzter Transport von Arsen- und Schwermetallionen in die Umgebung unmittelbar gestoppt.

- Durch den Ladungsaustausch und Abscheidungen an den Elektroden findet bereits eine teilweise Sanierung statt, deren Effizienz u. a. durch die Betriebszeit, die elektrische Spannung und ggf. durch das Austauschen der Elektroden kontrolliert und gesteuert werden kann.

- Der normale Betrieb auf einem kontaminierten Gelände kann quasi ungestört fortgefuihrt werden; umfangreiche Bodenaushubarbeiten sind nicht erforderlich. Die elektrokinetische Sicherung und Sanierung ist kostengünstig.

- Der Einsatz der Elektrokinese gewährleistet dazu einen weitgehend homogenen Schadstoffaustrag. Das Verfahren eignet sich besonders gut, um polare Schadstoffe, insbesondere Schwermetalle, aus Böden zu entfernen.

- Das Verfahren kann hervorragend für die Sanierung unter bebauten Flächen, wie in Wohngebieten, auf Industriegelände usw., eingesetzt werden.

- Das elektrokinetische Verfahren kann außer zur Sanierung von Altlasten auch zu Sicherungsmaßnahmen eingesetzt werden.
- Bei einer in-situ-Sanierung entfallen die hohen Kosten fuir die Auskofferung des belasteten Bodens, für seine Lagerung bzw. seinen Transport zu einer Bodenwaschanlage.

- Unter Ausnutzung der Elektroosmose kann durch eine genau definierte Anordnung der Elektroden die schadstoffbelastete Grundwasserströmung in bestimmte Richtungen gelenkt werden, z. B. zu einem Förderbrunnen für die Grundwassersanierung. Ein Abströmen in unerwünschte Richtungen, etwa zu einem Fließgewässer, kann verhindert werden.

- Die Ionen-Konzentrationen im Bereich der Elektroden werden z. T. so groß, dass die elektrokinetische Bodensanierung ggf. auch zur Rohstoffgewinnung im Sinne eines Recycling-Verfahrens eingesetzt werden kann.

- Eine geringe Permeabilität des Bodens bei diesem Verfahren wird überkompensiert durch die großen elektroosmotischen Feldstärken, die sich innerhalb der Doppelschicht zwischen Kornmatrix und Fluid einstellen. Die Feldstärken können lokal so groß sein, dass sie selbst fest adsorbierte Schadstoffe aus dem Feststoffverband herauslösen, d. h. entgegen der Erwartung mobilisieren können. Umgekehrt ist aber auch eine gezielte Immobilisierung möglich.

\subsection{Elektrokinetisches Verhalten und Trans- port von Schwermetallen und Arsen}

Die Literaturauswertung [2] ergab:

- Es gibt keine gesicherten Erkenntnisse über das elektrokinetische Verhalten und den Transport von Schwermetallen und Arsen, es sind lediglich Laborversuche mit Schwermetallen bekannt.

- An vielen Stellen wird uiber die Bedeutung der Arsendekontamination geschrieben, aber es wurden kaum Untersuchungen zum elektrokinetischen Verhalten von Arsen in Böden und auch zur Sanierung arsenkontaminierter Böden mit anderen Verfahren durchgefuihrt.

Dieser Widerspruch ist leicht zu erklären, wenn man sich mit der Arsenproblematik tiefgründiger auseinandersetzt. Die Schwierigkeiten liegen einerseits in der Giftigkeit vieler Arsenverbindungen, was einige besondere Vorsichtsmaßnahmen bei der Durchfuihrung der Experimente voraussetzt. Andererseits spielt der ampholyte Charakter des Arsens und dadurch eine starke Abhängigkeit der chemischen und Transporteigenschaften (und dabei entstehenden Arsenspezies) vom pH-Wert eine große Rolle, was die Experimente entscheidend verkompliziert. Darüber hinaus ist es schwierig, eine Substanz zu finden, die man als „Modellkontaminant“ anstelle der Arsenverbindungen in Simulationsversuchen einsetzen kann. Für Schwermetallkontaminanten sind diese Schwierigkeiten nicht so ausgeprägt, es gibt viele Modellsubstanzen, die sehr gut fuir solche Versuche geeignet sind (s. auch die Literaturauswertung in [1]).

\section{Ergebnisse}

Die Transportprozesse von Arsen im Boden unter Einwirkung des elektrischen Feldes lassen sich nicht mit be- 
kannten mathematischen Ansätzen beschreiben. Die Literaturauswertung ergab, dass alle mathematischen Modelle für den Schadstofftransport und hydrogeologische Transportprozesse im Boden nur Stoffbilanzen beinhalten, aber nicht die Energiebilanzen berücksichtigen, die bei elektrokinetischen Prozessen eine wichtige Rolle spielen. Weiterhin ergab das Literaturstudium, dass fast alle Untersuchungen zum elektrokinetischen Verhalten von Schwermetallen mit tonigen Böden bzw. mit Böden mit sehr hohem Tonanteil durchgefuihrt wurden. Die Übertragung der erzielten Ergebnisse und Erkenntnisse auf sandige Böden ist dadurch nicht gesichert bzw. nicht möglich.

Die Anwendung bekannter theoretischer Ansätze bezüglich elektrokinetischer Transportphänomene und Verhalten von Schwermetallen im elektrischen Feld im sandigen Boden musste erst untersucht werden, bevor man mit der Interpretation dieser Phänomene im Originalboden aus Wildau anfing. Zum Arsen-Transport in kontaminierten Böden existieren in der Literatur kaum gesicherte bzw. widersprïchliche Angaben, es ist auch nicht klar, als welche Spezies Arsen transportiert wird - in anionischer oder kationischer Form.

\subsection{Labor- und Technikumsversuche}

Die Klärung dieser Fragen erforderte zahlreiche Vorversuche mit Modellböden, die in ihrer Beschaffenheit dem Wildauer Boden entsprechen, und mit Modellkontaminanten, die in ihrem elektrokinetischen Verhalten erwartungsgemäß den Schwermetallen und Arsen ähnlich sind. Es wurde anschließend an die Laborversuche ein in-situ-Technikumsversuch als letzte Vorstufe zum Freilandversuch unter dem Gesichtspunkt der lokalen Bedingungen des Standortes Wildau durchgefuihrt. Der Technikumsversuch dient als Bindeglied der Maßstabsübertragung vom Laborversuch auf den Freilandversuch und kann unter dem Aspekt der realen Feldbedingungen betrachtet werden.

Sowohl durch die Laborversuche als auch im Technikum wurde die Möglichkeit des vertikalen Schadstofftransports aufgezeigt. Dabei kann ein großer Bereich des niederohmigen Grundwasserleiters auf ein einheitliches elektrisches Potenzial gebracht werden, das von außen über die Beeinflussung der Eh/pH-Bedingungen als As/ SM-anziehend oder As/SM-abstoßend eingeregelt werden kann. Durch die Labor- und Technikumsversuche wurde nachgewiesen, dass sich Arsen und Blei als Vertreter der Schwermetalle elektrokinetisch im BodenGrundwassersystem vertikal und horizontal transportieren lassen. Es wurde festgestellt, dass sich Arsen und seine Verbindungen sowohl anionisch als auch kationisch verhalten. Für Schadstoffe, die an der Bodenmatrix adsorbiert sind, ist eine sehr langfristige elektrokinetische Behandlung erforderlich. Durch den Einsatz biologisch leicht abbaubarer Säuren (z. B. Zitronensäure, Essigsäure) können die Schadstoffe mobilisiert werden. Ein wichtiges Ergebnis der Labor- und Technikumsversuche ist die daraus hervorgegangene Konzeption einer elektrokinetisch-adsorptiven Barriere, bestehend aus einer Brunnengalerie zur Aufnahme der Aktivkohleelektroden. Als Gegenelektrode zu den AktivkohleAnoden fungiert eine parallel zur Linie der Brunnen angeordnete Reihe von Stahlelektroden. Durch das so geschaltete elektrische Feld wird erreicht, dass es in seiner Wirkung auf die negativ geladenen Arsen-Spezies der Grundwasserfließrichtung entgegengesetzt gerichtet ist.

\subsection{Schlussbemerkung}

Als relevante Monitorparameter für das Testfeld Wildau haben sich erwiesen:

- elektrochemische Monitorparameter (der pH-Wert des Prozesswassers und das Redoxpotenzial in den Elektrodenbrunnen),

- elektrische Monitorparameter (die elektrische Spannung, das Elektrodenpotenzial),

- chemische Parameter (die Konzentration von Arsen und Blei im Grundwasser).

Eine Kontrolle der Temperatur und der Leitfähigkeit ist nur dann erforderlich, wenn diese Größen extreme Werte annehmen. Als begleitende Maßnahme hat die geophysikalische Erkundung einen hohen Stellenwert. Die kontinuierliche Aufzeichnung geophysikalischer Parameter wie Potenzial und Eigenpotenzial, spezifischer Widerstand und Aufladefähigkeit des Bodens ermöglicht die Kontrolle des Ablaufs der elektrokinetischen Sanierung im Sinne eines Monitorings zu jedem Zeitpunkt.

Standortbezogene Analysemethoden (Untersuchungen zur Elution und Mobilisierbarkeit von Schwermetallen, sequenzielle Extraktion) ergaben, dass das Wasser weder Blei noch Arsen in nennenswertem Maße eluiert. Salzsäure eluiert zunächst beide Elemente, die Eluierbarkeit von Arsen nimmt aber mit Zunahme der Bleikonzentration und der Anzahl der Durchläufe ab. Es wurde festgestellt, dass Blei die Eluierbarkeit von Arsen behindert.

Eine feldspezifische Modellierung des Schwermetalltransportes auf der Grundlage chemischer und elektrokinetischer Ansätze und eine raum-zeitliche Entwicklung der Schwermetallionenkonzentration im Testfeld lassen sich aus dem partiellen Differentialgleichungssystem ableiten. Die Simulationsberechnungen zum Wasserhaushalt nach dem Finite Differenzen-Verfahren zeigen, dass eine laterale und vertikale Wasserbewegung stattgefunden hat, die eine Austrocknung des Bodens im Bereich des Technikums Wildau verhindert. Die regelmäßige Beprobung der Bodenluft in der näheren Umgebung der Kathoden bzw. der in den Kathodenbrunnen stehenden Luft auf die Existenz von Arsenwasserstoff erfolgte unter Einsatz der direktanzeigenden Proberöhrchen von Dräger, die auch in die Bodenluftsonde eingesetzt werden können. Die Auswahl der standortspezifischen On-site-Laboranalytik erfolgte gemäß sämtlicher vorhandener Vorschriften (Bundes-Bodenschutzund Altlastenverordnung (BbodSchV) vom 12. Juli 1999), die fuir die As- und Pb-Analytik aus Bodenluft, Grundwasser oder Boden gelten. 


\section{Literaturverzeichnis}

[1] FuE-Verbundvorhaben „Erarbeitung eines Leitfadens fuir die Anwendung elektroinduzierter in-situ-Sanierungsverfahren für bebaute Altlastenstandorte“, gefördert durch das BMBF, Förderkennzeichen 1491103, 1. Zwischenbericht, Technische Fachhochschule Wildau, 1999

[2] Literaturstudie „Erfahrungen und Ergebnisse bei der Gefahrenbewertung sowie der Sanierung arsenkontaminierter Standorte im In- und Ausland“, Technische Fachhochschule Wildau, 1999

[3] Alloway, B. J. (Hg.): Schwermetalle in Böden: Analytik, Konzentrationen, Wechselwirkungen. Berlin: Springer, 1999

[4] Lippke, G.; Mang, V.; Kripendorf, A.: Arsenverbindungen in Böden: ökologische Aspekte. In: TerraTech 6 (1997) 2, S. $58-64$

[5] Riedel, F. N.; Eikmann, T.: Zum natürlichen Vorkommen von Arsen und seinen Verbindungen in Böden und Gesteinen. In: Wissenschaft und Umwelt (1986) 3/4, S. 108-117

[6] Riedel, N.; Michels, S.: Zum natürlichen Vorkommen von Arsen im Wasser. In: Wissenschaft und Umwelt (1987) 4, S. $210-215$

[7] Petrjanow-Sokolov, I. W. (Hg.): Bausteine der Erde. Bd. 2 Mangan - Zinn. Moskau: Mir, 1977

[8] Gmelin, L.: Gmelin-Handbuch der anorganischen Chemie. System - Nr. 17 Arsen. Verlag Chemie, 1952

[9] Gerhartz, W. (Hrsg.): Ullmann's Encyclopedia of Industrial Chemistry. Vol. 3. Weinheim: VCH-Verlags-Gesellschaft, 1985, S. 113-141

[10] Klockmann, F.; Ramdohr, P.: Klockmanns Lehrbuch der Mineralogie. 16. Aufl. Stuttgart: Enke, 1978

[11] Borho, M.: Arsenentfernung in Grundwasserwerken durch optimierte Kopplung von Oxidations- und Fällungs-, Flockungsverfahren: chemische Grundlagen und verfahrenstechnische Realisierung. Garching: Ges. zur Förderung des Lehrstuhls für Wasserguite- und Abfallwirtschaft der Technischen Universität München, 1996 (Berichte aus Wasserguite- und Abfallwirtschaft; 127)

[12] Driehaus, W.: Arsenentfernung mit Mangandioxid und Eisenhydroxid in der Trinkwasseraufbereitung. Düsseldorf: VDI, 1994 (Fortschritt-Berichte VDI: Reihe 15, Umwelttechnik; 133)

[13] Reddy, K. R.; Parupudi, U. S.; Devulapalli, S. N.: Effects of Soil Composition on the Removal of Chromium by Electrokinetics. In: Journal of Hazardous Materials 55 (1997) 1-3, S. 135-158

[14] Wild, A.: Umweltorientierte Bodenkunde: eine Einfuihrung. Heidelberg: Spektrum, 1995

[15] Gisi, U.: Bodenökologie. 2. Aufl. Stuttgart: Thieme, 1997

[16] Sposito, G.: Bodenchemie. Stuttgart: Enke, 1998

[17] Schlichtling, E.: Einfuihrung in die Bodenkunde. 2. Aufl. Hamburg: Parey, 1986

[18] Schinner, F.; Sonnleitner, R.: Bodenökologie: Mikrobiologie und Bodenenzymatik. Bd. 4: Anorganische Schadstoffe. Berlin: Springer, 1997

[19] FuE-Verbundvorhaben „Erarbeitung eines Leitfadens für die Anwendung elektroinduzierter in-situ Sanierungsverfahren für bebaute Altlastenstandorte“, gefördert durch das BMBF, Förderkennzeichen 1491103, Abschlussbericht, Technische Fachhochschule Wildau, 2000
[20] Goldmann, T.: Elektrokinetische Bodensanierung: Erfahrungen in Labor und Technikum. In: Kiefer, K.-W. (Hg.): Grundwasserschadensfälle durch Bodenkontaminationen: Überwachungssysteme, Untersuchungsmethoden, Sanierungsverfahren. Taunusstein: Blottner, 1996, S. 169-190

[21] Ernst, W.H.O.: Wirkung erhöhter Bodengehalte an Arsen, Blei Cadmium auf Pflanzen. In: Kreysa, G. (Hg.): Beurteilung von Schwermetallen in Böden von Ballungsgebieten: Arsen, Blei und Cadmium; internationale Expertenbeiträge und Resümee der DECHEMA-Arbeitsgruppe „Bewertung von Gefährdungspotentialen im Bodenschutz“. Frankfurt am Main, 1994

[22] Hiller, D.: Elektronenmikrostrahlanalysen zur Erfassung der Schwermetallbindungsformen in Böden unterschiedlicher Schwermetallbelastung. In: Mitteilungen der deutschen Bodenkundlichen Gesellschaft 66 (1991) (2), S. $1085-1088$

[23] Kersten, M.; Förstner, U.: Speciation of Trace Elements in Sediments. In: Batley, G. E. (Hg.): Trace Element Speciation: Analytical Methods and Problems. CRC Press, 1989, S. 245

[24] Rahner, D.; Grünzig, H.; Ludwig, G.: Studie zur elektrochemischen Sanierung kontaminierter Böden. In: TerraTech 4 (1995), S. 58-61

[25] Jehring, H.: Untersuchungen und Vorstellungen zu Möglichkeiten, Grenzen und Gefahren elektrochemischer Bodenbehandlung. Fachgespräch „Verfahren zur elektrochemischer Sanierung von Altstandorten“ am 22.09.1994 im Umweltbundesamt

[26] Matthess, G.: In-situ Treatment of Arsenic Contaminated Groundwater. In: The Science of a Total Environment 21 (1981), S. 91-104

[27] Jehring, H.; Jehring, R.; Rohde, R.: Elektrokinetische Sanierung kontaminierter Böden. In: Altlastensanierung '93. Vierter Internationaler KfK/TNO Kongreß über Altlastensanierung, 3.-7. Mai, 1993, Berlin, S. 1437-1438

[28] Goldmann, T.: Entwicklung der Verfahrenstechnik zur elektrokinetischen Sanierung von Böden, Abschlussbericht zum BMWI-Forschungsprojekt 631/93; Institut für Technologie und Umweltschutz e.V., Berlin, 1993

\section{Autoren}

\section{Prof. Dr.-Ing. Udo Hellwig \\ Dr. rer. nat. Olga Böhme \\ Dipl.-Geophys. Edgar Wetzig}

Technische Fachhochschule Wildau

Fachbereich Ingenieurwesen/Wirtschaftsingenieurwesen Tel. (0 33 75) 508-170

E-Mail: hellwigu@vt.tfh-wildau.de

\section{Priv.-Doz. Dr. habil. Werner Schmitz}

Universität Leipzig

Fakultät für Chemie

Institut für Mineralogie, Kristallographie und Materialwissenschaft

Scharnhorststraße 20, 04275 Leipzig

Tel. (03 41) 9736266

E-Mail: schmitz@rz.uni-leipzig.de

*ITVA: Ingenieurtechnischer Verband Altlasten e. V. 